\title{
Análisis de Experiencias en la Promoción de Actividad Física
}

\section{Beatriz Mena-Bejarano}

Fisioterapeuta. M. Sc. Discapacidad e Inclusión Social. Esp. Ejercicio Físico para la Salud. Departamento del Movimiento Corporal Humano y sus Desordenes. Universidad Nacional de Colombia. E-mail: bmenab@unal.edu.co

Recibido 12 Mayo 2006/Enviado para Modificación 15 Junio 2006/Aceptado 7 Julio 2006

\section{RESUMEN}

Objetivo Este artículo presenta las fortalezas y debilidades de algunas experiencias de Bogotá, de promoción de la salud, con énfasis en actividad física.

Métodos Como proceso inicial se realizó una revisión documental de los modelos teóricos de intervención y de los lineamientos propuestos en declaraciones Internacionales y Nacionales relacionadas con la actividad física. El análisis se desarrolló en tres fases: 1) Fase exploratoria. Se recolectó información general de los programas de actividad física existentes, encaminados a la prevención de enfermedades crónicas no transmisibles (ECNT) y a la promoción de la salud y se realizó la revisión bibliográfica 2) Fase Descriptiva. Se caracterizaron los programas de promoción de la actividad física según sus objetivos, alcances, estrategias utilizadas y las metodologías inmersas en los procesos y 3) Fase Analítica. Se realizó el análisis crítico de los programas existentes a la luz de las categorías establecidas por la autora para hacer una aproximación a las fortalezas y debilidades de los mismos.

Resultados El análisis permitió destacar aspectos favorables (efectivos) y desfavorables de los programas de actividad física para ser tenidos en cuenta en futuras propuestas de intervención basadas en actividad física. Los programas analizados promueven la autogestión y autonomía mediante la generación de redes de apoyo y la capacitación a líderes para garantizar su sostenibilidad.

Conclusiones En general, estos programas intervienen a la persona y a la comunidad a través de la realización de talleres tanto prácticos como teóricos pero no cubren todo el proceso vital humano, sino que se concentran en grupos poblacionales específicos. Tienen deficiencia de divulgación en medios masivos de comunicación pues aún no han implementado estrategias comunicativas masivas y no responden a políticas claras dentro de cada institución. 
Palabras Clave: Promoción de la salud, enfermedades crónicas no transmisibles, actividad física, modelos de intervención. (Fuente: DeCS, BIREME).

\section{ABSTRACT \\ Experiences in promoting physical activity}

Objective Evaluating the strengths and weaknesses of some experiences emphasising physical activity in promoting health in Bogotá.

Methods A documentary review of theoretical models of intervention was first undertaken, followed by examining guidelines proposed in international declarations and in Bogotá related to physical activity. Three-phase analysis was carried. 1) The exploratory phase involved collecting general information regarding programmes pertaining to physical activity aimed at preventing non-transmittable chronic diseases (NTCD) and promoting health. 2) The descriptive phase involved characterising programmes for promoting physical activity according to their objectives, scope and the strategies and methodologies used in such processes. 3) The analytical phase involved a critical analysis of current programmes in terms of their strengths and weaknesses.

Results The programmes analysed here promoted self-management and autonomy by producing support networks and training leaders for guaranteeing their sustainability.

Conclusions The programmes involved people and the community by holding practical and theoretical workshops which did not cover the whole vital human process, but concentrated on specific population groups. They were deficient in broadcasting in the mass-media.

Key Words: Physical activity, model, intervention, non-transmittable chronic diseases (source: DeCS, BIREME).

L

a literatura científica ha documentado en forma extensa los beneficios de la actividad física para la salud y la calidad de vida. La evidencia científica ha documentado su importancia en la reducción del riesgo de morir por enfermedades cardiovasculares, en el control del sobrepeso, la obesidad, la diabetes mellitas tipo II y el cáncer de colón. (1-4) Como resultado de los procesos investigativos y de experiencias diversas se han proclamado múltiples declaraciones, programas, normas y principios en beneficio de la población en general con respecto a la importancia de la actividad física, el deporte y la recreación como elementos fundamentales para la promoción de la salud y la prevención de la enfermedad. Diversas entidades internacionales como la Federación Internacional de Medicina del Deporte (FIMS), la Red de Actividad Física de las Américas (RAFA), la Organización Mundial de la Salud (OMS), el Center for Disease Control and Preven- 
tion (CDC) y la Declaración de Sao Paulo para promover la actividad física en el mundo, han reconocido que la práctica regular de actividad física es uno de los principales factores protectores en la prevención de las ECNT. Desde esta perspectiva, en los últimos años han surgido diversos programas de promoción y prevención con enfoque teóricos diversos.(5-8)

Existen diversas clasificaciones de los enfoques utilizados para la realización de programas de promoción de la actividad física. La clasificación del Physical Activity and Health, a Report of The Surgeon General (9), reconocida internacionalmente propone los siguientes modelos de intervención. En primer lugar, los Modelos de Intervención Individual que enfatizan la intervención en la persona a la cual están dirigidas las acciones. Tienen en cuenta el grado de motivación, percepción de riesgos o actitud hacia la actividad física. Dentro de esta categoría se destacan, el Modelo Transteórico de Comportamiento, el Modelo de Procesamiento de la Información, el Modelo de Prevención de Recaídas y el Modelo de Creencias en Salud. En segundo lugar, los Modelos de Intervención Interpersonales, que incluye aquellos programas que enfatizan la intervención en grupos de personas a la cual están dirigidas las acciones. Los mas conocidos son, La Teoría del Aprendizaje Social o Teoría cognitiva social, la Teoría de Acción Razonada, la Teoría del Comportamiento Planeado y el Apoyo Social. En tercer lugar, están los Modelos de Intervención Comunitarios, que plantean que las intervenciones para ser efectivas deben influir en múltiples niveles porque la salud está condicionada por muchos subsistemas ambientales, que incluyen la familia, la comunidad, el lugar de trabajo, las creencias y tradiciones, las finanzas y los entornos físicos y sociales. En esta categoría se encuentran, La Teoría de la Difusión de Innovaciones, el Modelo Ecológico, el Modelo de Organización de la Comunidad y la Teoría del Cambio Organizacional.(10)

El objetivo de este estudio fue documentar, con base en los enfoques teóricos y las declaraciones internacionales, los programas de actividad física desarrollados en Bogotá, dirigidos a reducir el sedentarismo y prevenir enfermedades crónicas no transmisibles; con el propósito de retomar los elementos exitosos para estudios futuros relacionados con el tema.

\section{MÉTODOS}

En la fase exploratoria se realizó la búsqueda de programas de promoción de la salud y prevención de enfermedades crónicas no transmisibles (ECNT) encaminada a promover la actividad física. Esta fase se inició a través de un acercamiento a Instituciones públicas y privadas, que desarrollan programas de actividad física en Bogotá, tales como el Instituto Distrital para la Re- 
creación y el Deporte (IDRD), la Secretarías Distrital de Salud (SDS), la Secretaría de Educación del Distrito (SED), algunas Cajas de Compensación, la Asociación de Medicina del Deporte (AMEDCO) y el Departamento Administrativo de Bienestar Social (DABS). El objetivo fue conocer los programas que desarrollan, sus principales características y su impacto.

Por medio de la revisión documental, y la comunicación directa (correo electrónico, teléfono y fax) con los coordinadores de los programas, se logró identificar los fundamentos de los programas en las tres regiones y aspectos claves relacionados con las características, procesos, metodologías, resultados y problemas de los programas considerados. Para complementar este análisis se desarrollaron en las tres regiones de estudio, talleres y entrevistas semiestructuradas para obtener información relevante.

En Bogotá se revisaron las páginas Web y los documentos e informes de los Programas de entidades distritales entre las que se cuentan: El Instituto Distrital para la Recreación y el Deporte (IDRD), El Departamento Administrativo de Juntas de Acción Comunal (DAJAC) y el DABS. Durante el primer trimestre de 2004, se revisaron cuatro experiencias de programas de actividad física: el "Programa Salud y Calidad de Vida" en la localidad de Engativa, el programa "Muévete Bogotá" y el "Programa Tercera Edad y Atención al Discapacitado” ambos, del Instituto Distrital para la Recreación y el Deporte (IDRD) y el de "Proyectos y modelos de políticas de juventud e intervención” del Departamento Administrativo de Acción Comunal (DAAC).

Se realizaron entrevistas a directores y coordinadores de los programas, orientadas a identificar su correspondencia parcial, total o mixta con los modelos teóricos y a consolidar información relevante con base en las siguientes categorías: (a) Identificación. Que incluye información relacionada con la denominación de la experiencia o programa, oficina o dependencia a cargo del programa, funcionarios a cargo del programa, población a la cual se dirige y algunos aspectos generales como la descripción del proceso de creación y gestación de la idea; (b) Diseño. Se refiere a las etapas o fases del programa y las actividades realizadas en cada una de ellas. Se contemplaron aspectos particulares como el enfoque teórico que sustentaba los programas, la aprobación de la propuesta, financiación, asignación de responsabilidades para ejecutar el programa, organización del programa, entre otros; (c) Implementación. Considera las estrategias utilizadas para la promoción de la salud y la prevención de las enfermedades crónicas no transmisibles (ECNT); y (d) Evaluación. Determinando los proceso de seguimiento y control del programa. 
REVISTA DE SALUD PÚBLICA • Volumen 8 (Sup. 2), Noviembre 2006

\section{RESULTADOS}

Generalidades de los Programas en Bogotá

El Programa Muévete Bogotá (MB): desarrollado por el IDRD en Bogotá, se considera uno de los principales programas en el ámbito local, regional y nacional, al punto que ha logrado reconocimiento internacional (4). Este programa ha sido entendido como "una estrategia pedagógica que busca generar cambio de hábitos en la población” hacia la práctica regular de actividad física, utilizando las actividades de la vida diaria, los ratos de ocio y el tiempo libre como espacios fundamentales para su desarrollo, con el fin de incidir positivamente en el nivel de salud de las personas al tiempo que plantea una solución alternativa a los factores adversos que conllevan al sedentarismo sin ocasionar modificaciones drásticas en las rutinas diarias de los individuos; es decir plantea como estrategia la modificación a estilos de vida activos.

Este programa, pretende incrementar la actividad física en diversos ambientes (la escuela y el trabajo) por medio de dos subprogramas denominados: "Muévete Escolar” y "Empresas Amigas”. Muévete Escolar busca que se adquiera el hábito de la actividad física diaria, con especial énfasis en la comunidad educativa (niños, docentes y padres de familia), involucrando acciones que permitan tener una población futura sana y disminuir los riesgos de ECNT. Utiliza estrategias lúdicas llamativas como publicaciones, festivales y dramatizaciones alusivas al tema. Las Empresas Amigas son aquellas entidades, instituciones, asociaciones, grupos de trabajo de tipo laboral, educativo, comunitario o de salud, entre otras, que deseen promover programas de actividad física a usuarios, trabajadores, estudiantes, miembros, familias, trabajando conjuntamente con Muévete Bogota en lograr la disminución del sedentarismo.

El programas ha obtenido beneficios económicos asociados con ahorro en costos médicos cuando se invierte en equipos y tiempo para la actividad, mayor productividad laboral, menor ausentismo, mayor rendimiento académico en estudiantes, disminución de enfermedades y riesgos profesionales, mayor bienestar y placer de funcionarios, niños, docentes asegurando que la actividad física siempre es una política en al que ganan todos. Adicionalmente Muévete Bogotá realiza eventos masivos de promoción y divulgación del programa y de la actividad física mediante la implementación de actividades que evalúan y diagnostican la condición física de las personas.

El Programa Salud y Calidad de Vida de la Localidad de Engativa: Es una iniciativa que surge como resultado de un convenio institucional entre la 
Alcaldía Local de Engativa y la Universidad Nacional de Colombia. La idea inicial surge por las necesidades de la localidad expuestas en el Plan de Desarrollo, el cual plantea como un problema importante la no-atención o la atención insuficiente de los problemas derivados de las Enfermedades Crónicas no Trasmisibles (ECNT) cuyas cargas se le asignan a la familia sin que en la mayoría de casos exista la posibilidad de reaccionar adecuadamente. Este proyecto se dirigió a poblaciones específicas las cuales fueron catalogadas como: pacientes Tipo I sin diagnóstico de Enfermedad Crónica no Transmisible (ECNT), pacientes tipo II: con diagnóstico de ECNT y tipo III con discapacidad secundaria a una ECNT.

Para la ejecución del proyecto se plantearon tres fases. La primera fase denominada de evaluación e implementación, consistió en el diseño de dos instrumentos de medición. El Formato de Valoración de Actividad Física, dirigido a los pacientes tipo I, se compone de dos partes la primera suministra información demográfica de la población y la segunda, determina el grado de actividad física gracias a la encuesta IPAQ y a específicos de resistencia cardiovascular, fuerza y flexibilidad, aspectos personales de motivación, gustos y preferencias. Para los pacientes tipo II y III se diseñó un formato de Evaluación Fisioterapéutica que implicaba una visita domiciliaria. El proyecto parte de un supuesto de mejoría, que asume que la mayoría de las personas son sedentarias (aunque esta información no este confirmada) y que están expuestas a algún factor de riesgo para adquirir ECNT. Con base en estas premisas se plantean tres objetivos: 1) Disminuir la adquisición de los factores de riesgo, 2) volver activas a las personas que son sedentarias y 3) Convertir la Actividad Física en factor protector de ECNT.

Durante la segunda fase denominada de intervención, se clasificaron los individuos en sedentarios, irregulares y activos, y los diferentes resultados se relacionaron desde el aspecto motivacional, sociocultural y físico, para realizar acciones más interdisciplinarias, orientadas a promover la actividad física en forma personalizada según su condición específica.

Los psicólogos analizaron aspectos motivacionales, correspondientes a estados de cambio, gustos y preferencias, para determinar qué aspectos llamaban más la atención del individuo para realizar actividad física. Igualmente al combinar la motivación, las barreras, los gustos y preferencias fue posible establecer los aspectos socioculturales que podían facilitar la adherencia al programa de Actividad Física. De este análisis surgió una gama de posibilidades, que oscilaba entre estilo de vida activo, actividades recreativas y deporte. 
En el aspecto sociocultural, las modalidades de trabajo utilizadas ayudaron a romper algunos mitos relacionados con la práctica de Ejercicio Físico. Las representaciones sociales de actividad física iniciales de relacionaban con "ejercicios fuertes que lleven a sudoración y cansancio extremo", "aeróbicos", "trotar", "practica de algún deporte", "ir al gimnasio", entre otros. Esta propuesta de trabajo intentó modificar el imaginario de que la práctica de actividad física sólo involucra el aspecto físico (biológico), sino también el ambiente social y el cultural.

Paralelo a la evaluación, se implementaron prácticas de actividad física en 17 barrios de la localidad (tres veces a la semana en sesiones de una hora y media), con el objetivo no sólo de promover la actividad física como "deporte" o "ejercicio", sino para ofrecer una concepción más amplia de actividad física como medio que permite a través del cuerpo conseguir sustento, trasladarse, auto cuidarse, relajarse, comunicarse, explorar su cuerpo y conocer el mundo, y además permitir una comprensión de la salud no solo física, sino también mental y social. Todos los talleres se acompañaron de charlas educativas teóricas relacionadas con toma de signos vitales, flexibilidad, fuerza, corporalidad, potencia, y propiocepción.

El Programa Tercera Edad y Atención al Discapacitado del IDRD busca "que los adultos mayores estén activos, sean autónomos, mejoren su calidad de vida, su estado de salud, y que principalmente los involucren en actividades sociales donde se integren, socialicen y participen como ciudadanos útiles a toda nuestra sociedad”. El programa empezó en 1998 y la idea surgió por necesidades sentidas de la comunidad. Uno de sus objetivos de este programa es garantizar su permanencia en las Localidades, que no dependan de la contratación de personal externo a la comunidad; por esta razón el programa se ha modificado de acuerdo a las necesidades del grupo. En la actualidad los y las adultas mayores se han apropiado de sus procesos y buscado más asesoría y apoyo, al punto que también trabajan con otras entidades como el Departamento Administrativo de Bienestar Social (DABS), las Alcaldías locales y el Instituto Distrital de Cultura y Turismo. Este programa da a conocer los beneficios de la práctica regular de AF en tercera edad con relación a la condición de salud y calidad de vida, para disminuir el sedentarismo y volverse activos. La estrategia utilizada es formarlos como multiplicadores. Inicialmente se vivencian los beneficios, para poder replicarlos en sus casas, en su comunidad y de esta forma ser protagonistas de su propia acción. Las acciones prioritarias para la promoción de la AF son de índole recreativa y cultural, las que además de realizar AF, afianzan valores y tradiciones. Las mas utilizadas son la gimnasia de mantenimiento, Canita al juego, Danzas, Vivencias de antaño (de integración intergeneracional). 
Los "Proyectos y modelos de políticas de juventud e intervención” del DAAC: Utilizan como modalidad de trabajo el desarrollo, a partir de los consejos de juventud, de asesorias a los alcaldes locales en lo relacionado con la recreación y el esparcimiento, priorizando a la actividad física en la juventud. Aunque estos proyectos no realizan acciones encaminadas exclusivamente hacia la actividad física, si la promueven y contemplan los ambientes. De los procesos generados han surgido decisiones de las alcaldías locales que se encaminen a la puesta en marcha de programas centrados en actividad física pero siempre vinculados a otras dimensiones del trabajo de dichos consejos. Si bien la actividad física como tal no es lo que fundamenta el trabajo del DAAC, en las acciones que se desarrollan a nivel local y barrial se evidencia un fuerte trabajo de esta. Las comunidades resuelven en conjunto hacer campeonatos deportivos en las instalaciones de los salones comunales, aeróbicos y programas para el adulto mayor. Esto lo desarrollan a nivel de Alcaldías Locales, DABS, el IDRD, la Secretaría de Educación, Instituto Distrital de Cultura y turismo (IDCT), el Departamento Administrativo del medio Ambiente (DAMA), la Secretaría de Salud de Bogotá, entre otras con 11 entidades en total.

De esta experiencia es importante rescatar dos aspectos, el primero, se ha venido desarrollando un trabajo con relación al cuerpo y el manejo del cuerpo entendido como un ente de relación con el entorno, lo cual implica la práctica regular de $\mathrm{AF}$ y este proceso se ha mantenido aproximadamente durante 14 años a pesar de los cambios en las políticas de gobierno, en los gobernantes y de la ausencia de una política local desde el Departamento relacionada con el fomento de la AF en la juventud. Se puede decir que es un programa que la incentiva en forma tácita y que promueve la participación comunitaria.

\section{Análisis Crítico de los Programas}

Se tuvieron en cuenta las características generales que debe tener un programa de promoción de la Actividad física enmarcada en una política pública, lo que permitió establecer las siguientes categorías de estudio:

1. Existencia de un ambiente social y físico que conduzca a la adopción y mantenimiento de estilos de vida físicamente activos (11). Las Entidades del Estado han implementado ambientes físicos cada vez más favorables para la práctica regular de AF como las ciclo-vías, las ciclo- rutas, la modificación y creación de parques. Las Juntas de Acción Comunal promueven la práctica de AF mediante actividades lúdicas y recreativas como por ejemplo los aeróbicos. En cuanto al ambiente social, la mayoría de programas se encami- 
nan a lograr autogestión y autonomía mediante la generación de redes de apoyo que garanticen la continuidad de los mismos sin la intervención obligada de las instituciones.

En el caso particular del Programa Tercera Edad y Atención al Discapacitado del IDRD se cuenta con una red en la cual se tienen diversos grupos de adultos mayores que están afiliados o asociados con el IDRD; para constituir estas redes se designan líderes que se capacitan gradualmente en diversos temas: la AF y su importancia en la salud, principios del entrenamiento y salud en general. De igual manera Muévete Bogotá capacita a los gestores que se harán cargo del programa a nivel escolar o empresarial. Ellos asumen el rol de multiplicadores y posteriormente difunden los conocimientos adquiridos a sus respectivos grupos. La idea es brindar asesoría a estos promotores para que tengan buenas bases conceptuales pues trabajan con población de alto riesgo.

2. La Intersectorialidad. Esta se concibe como el trabajo conjunto de diferentes sectores para lograr la gestión y promoción de actividad física, entre los que se cuentan salud, deporte, educación, cultura, medios de comunicación / información, planificación urbana, transporte, gobierno local /municipalidades, planificación financiera y económica(OMS, 2002). Aunque los programas analizados responden a un interés gubernamental específico para promover estilos de vida saludable, se evidencia que son programas desagregados e independientes en los cuales es difícil definir puntos de "encuentro" específicos. Cada representante responde por el Programa institucional, sin embargo la cooperación interinstitucional no es evidente. No obstante, el programa del DAAC podría ser la excepción pero la información obtenida a través de las fuentes orales es insuficiente para establecer con certeza si existe trabajo multisectorial o intersectorial.

3. Enfoque teórico que sustenta los programas. La mayoría de programas tienen enfoques individuales e interpersonales más que comunitarios y consideran en forma secundaria el entorno y las condiciones de vida de la persona y de la familia. En general todos los programas que intervienen a la persona y a la comunidad, no lo hacen a través de todo el proceso vital humano, sino que se concentran en grupos poblacionales específicos. Se encuentran experiencias dirigidas únicamente al grupo de tercera edad, a las personas con discapacidad, a las personas con ECNT o a los trabajadores. No existen programas de intervención masiva que involucren a toda la población. 
4. Existencia de Programación y planificación en los programas de actividad física (11). La mayoría de los programas se ejecutan a través de tres fases: 1) Evaluación inicial y diagnóstico, 2) Implementación y 3) Seguimiento y control. Sin embargo ninguno de estos cuenta con información sistematizada que permita determinar el impacto a mediano y largo plazo. Todos los programas presentan mayor desarrollo conceptual y metodológico de la fase I de Evaluación Inicial y Diagnóstico con relación a las otras. El Programa de la localidad de Engativá, realizó un estudio epidemiológico de la localidad antes de implementar el programa mediante la aplicación de la encuesta de condiciones de vida, que aporta información similar a la encuesta de hogares del Departamento Administrativo Nacional de Estadística (DANE). De igual forma se identificaron los factores de riesgo mediante la aplicación de pruebas de laboratorio clínico para contar con información objetiva que permitiera establecer la efectividad del programa. Por otra parte, en "Muévete Bogotá" se realiza un proceso evaluativo que determina, según el modelo transteórico, el estado comportamental de los participantes, la condición física y realiza las encuestas para determinar nivel de AF y riesgo cardiovascular (IPAQ y PARQ \& YOU respectivamente).

5. Capacidad de respuesta a políticas de participación social. Desde la perspectiva de organización social se entiende como aquella, "dirigida a consolidar procesos de larga duración, en los cuales se habilita a la comunidad para que desarrolle destrezas y capacidades para tomar decisiones que van desde la identificación de problemas y necesidades, la articulación de expectativas, la formulación hasta la negociación de propuestas de solución" (12). Los cuatro programas intentan garantizar la continuidad en el tiempo mediante la identificación de líderes. En el grupo de tercera edad en el momento del estudio se tenían 7 liderazgos en pro de que esta población sea cada vez más independiente. Por otro lado aunque en el Programa "Proyectos y modelos de políticas de juventud e intervención" es claro que la promoción de la AF como hábito saludable de vida no es el objetivo primordial, se permite dentro de la organización social, una acción de los colectivos comunitarios. Los alcances en la participación social se consiguen en forma incipiente porque la iniciativa y las estrategias para lograr empoderamiento surgen más desde el programa mismo que desde la comunidad.

6. Integralidad de los Programas. Aunque la literatura reporta que el éxito de estos programas depende de la intervención integral en todas las dimensiones del individuo, ya sea física, emocional o ambiental, los programas no son abordados en forma holística (12). Sin embargo, algunos programas cuentan con la presencia de un equipo interdisciplinario conformado por profesionales de diversas áreas como medicina, psicología, nutrición y 
dietética, fisioterapia, educación física, entre otras, lo cual garantiza en forma parcial, el logro de este objetivo. En cuanto a los niveles de intervención, casi todos los programas se inscriben en el nivel personal e interpersonal. Por otra parte, no se evidencia una articulación ni vertical ni horizontal con la estructura política del estado. (Nacional, regional, local y comunitario)

7. Orientación Integral de Recursos. Los recursos se han dirigido más a acciones encaminadas a la promoción y a la prevención. En Bogotá, los recursos de cada programa dependen del sector que lo desarrolla, es decir, salud o recreación y deporte.

8. Existencia de base científica para definir, diseñar y realizar las actividades del programa. Todos los programas responden a los lineamientos nacionales e internacionales establecidos sobre AF y se fundamentan en avances teóricos recientes. Algunos de ellos se basan en información primaria de estudios, encuestas, etc., obtenida directamente por o para el programa.

Las fortalezas de los programas

El objetivo de esta fase es extractar los aspectos positivos que se deben rescatar de estas experiencias con el fin de tenerlas en cuenta para futuros programas de promoción de la salud a través de la Actividad Física. Se exponen en forma global las implicaciones y los retos a los que nos vemos enfrentados para garantizar el éxito de estas intervenciones.

Se pueden concluir que los cuatro programas, promueven la autogestión y autonomía mediante la generación de redes de apoyo y la capacitación a líderes para garantizar la sostenibilidad de los programas. Muévete Bogotá del IDRD se considera uno de los principales programas macro en el ámbito regional y nacional, al punto que los esfuerzos continuos en la planificación, ejecución y evaluación del mismo se reflejan en el reconocimiento que este programa ha tenido no sólo nacional sino también internacionalmente. Como estrategia motivacional utiliza la entrega de incentivos y premios a los participantes en los eventos como: llaveros, lazos, cartas, etc. El enfoque de este programa es individual.

Salud y Calidad de Vida de la Localidad de Negativa ofrece una práctica multi-opcional de actividad física. Mediante la educación muestra como la actividad física tiene diversas posibilidades: 1)Estilos de Vida Activos, 2) Cambios desde la práctica de algún deporte y 3) Cambios desde la Recreación y el uso del tiempo libre. Por otra parte, la intervención estuvo a cargo 
de un equipo interdisciplinario relacionando aspectos motivacionales, socioculturales y físicos.

Tercera Edad y Atención al Discapacitado del IDRD. Tiene en cuenta la participación social activa de las personas de tercera edad en los procesos de autogestión del programa, buscando autonomía tanto del programa como de los participantes. Pretende lograr un cambio de la visión del viejo en su entorno familiar (transmite cultura y vitalidad) y rescatar juegos tradicionales para involucrarlos en la actividad física. Su principal fortaleza es la consolidación de una red de grupos de adultos mayores y la presencia de líderes capacitados para la práctica adecuada de AF. A nivel local debido al contacto con las alcaldías, trabaja con la colaboración de diversas instituciones distritales. Es decir, existe trabajo interinstitucional.

Proyectos y Modelos de Políticas de Juventud e Intervención del DAAC. Como principales fortalezas se encuentran, la documentación del proceso, la existencia de material escrito y la sostenibilidad (14 años). Las convocatorias de estos programas involucran a los consejos locales de juventud, las organizaciones juveniles organizadas y no organizados mediante diversas estrategias: 1) La comunicación. Que se ha dado con la página en Internet "Bogotá joven”. (2) La creación de una red de lazos jóvenes que se llama "relajo” y 3) la realización de talleres de socialización donde exponen la política y los mismos jóvenes identifican sus necesidades según cada localidad. Todas estas estrategias están encaminadas a difundir la política invitando a la participación, que permite que el proceso más que crítico sea propositivo pues los mismos jóvenes intentan dar soluciones a sus necesidades. Un reflejo de este esfuerzo es que ya se realizó un seminario denominado "una política de juventud para Bogotá”.

Las debilidades de los programas

En general todos los programas que intervienen a la persona y a la comunidad, no cubren todo el proceso vital humano, sino que se concentran en grupos poblacionales específicos, como son las personas de tercera edad, los escolares, las personas con discapacidad y los trabajadores.

Ausencia de un abordaje educativo del sedentarismo, que se acerque a la construcción histórica de sus causas, y no se limite a la realización de talleres tanto prácticos como teóricos, de modo que trascienda la cotidianidad de la práctica de Actividad Física y posibilite una real transformación de los estilos de vida en las personas. 
Existe deficiencia de divulgación en medios masivos de comunicación y no se han implementado estrategias comunicativas que sensibilicen a las comunidades a cerca del riesgo del sedentarismo ni las relacionadas con el fomento de la práctica regular de Actividad Física.

La puesta en marcha de los programas y su éxito depende en forma marcada de la voluntad política y conciencia en las Directivas de las Instituciones de turno, ya que no responden a políticas claras dentro de cada institución. Aunque el Ministerio de Salud propuso dentro de las principales estrategias acogidas como elementos fundamentales para el desarrollo del plan de actividades, la coordinación intersectorial, la educación, la comunicación, la asistencia individual, la evaluación y la participación comunitaria, ninguno de estos programas desarrollan en forma integral estas estrategias (5).

\section{DISCUSIÓN}

En términos generales, se puede decir que los programas existentes responden de manera parcial a los lineamientos planteados internacionalmente e implementan acciones que propenden por la autogestión y el auto cuidado, por medio de la identificación de líderes y promotores en salud. Sin embargo, aún no se ha logrado un trabajo interinstitucional que permita la contemplación de factores personales, ambientales, socio-económicos que propicie una verdadera movilización social, cuyo objetivo es "desarrollar un conjunto de acciones que le permitan al Estado, los gobiernos locales, y las comunidades, además de la concertación de acciones, el establecimiento de alianzas y consolidación de redes entre los diferentes grupos involucrados para desarrollar acciones más eficientes y alcanzar metas de mayor impacto social" (12-13).

Se destaca que estos programas constituyen experiencias relativamente recientes que están en proceso de consolidación y evaluación de impacto. Están bien estructurados y responden a diagnósticos poblacionales con relación al tema del sedentarismo, para luego actuar con estrategias y actividades pertinentes dirigidas a fomentar la práctica regular de AF. Por tal razón, los programas hasta ahora están avanzando en la construcción de indicadores y procesos de seguimiento a la gestión y evaluación del impacto, lo cual en este momento es su mayor debilidad. Debido a la escasa sistematización y acceso a la información no fue posible documentar las experiencias en forma mas profunda.

Todos los programas analizados responden a un interés gubernamental específico para promover estilos de vida saludable. Sin embargo, se eviden- 
cia que son programas desagregados e independientes en el sentido que cada uno de ellos responde a un programa institucional específico, que obedece a dinámicas de racionalización de recursos institucionales. Este aspecto confirma que aún no se ha logrado la coordinación intersectorial y la participación comunitaria, aspectos que requieren la articulación de esfuerzos intersectoriales de cultura, educación, salud y planeación Distrital, entre otros.

Si bien no existe una política pública nacional de promoción de la AF para prevenir el sedentarismo recientemente en Bogotá, durante las tres últimas Alcaldías populares, se ha creado un contexto que ha permitido una incorporación tácita de acciones para el fomento de la AF, ejemplo de esto, es el programa Muévete Bogotá, que ha logrado legitimar el tema de AF y generar la reflexión. Los y las habitantes de Bogotá han logrado un mayor conocimiento y apropiación del programa. Entre los principales logros que se pueden destacar esta la recuperación del espacio público, que incluye adecuación y mantenimiento de zonas verdes y parques para la recreación y el aprovechamiento del tiempo libre, la construcción de ciclorutas, la asignación de recursos para la ejecución de programas para mejorar la calidad de vida de los ciudadanos, entre otros. En cuanto a las estrategias que han utilizado con mayor frecuencia estos programas son la capacitación, educación, asistencia individual y evaluación. En un grado mucho menor la comunicación y la participación comunitaria.

El análisis de estas experiencias llevó a reiterar que los programas de promoción de la actividad física deben integrarse en la vida diaria de las personas y promoverse en todos los grupos sociales, desarrollando entornos propicios que permitan el acceso a los mismos, a partir de estrategias de intervención como: a) comunicación (campañas masivas y avisos en puntos estratégicos, b) Accesos sociales y comportamentales mediante programas individuales para propiciar cambios comportamentales y de salud e intervenciones de soporte social en la comunidad y c) Creación de espacios y acceso a lugares para la actividad física. Así mismo, destacan la importancia que tienen los proyectos comunitarios organizados con componentes múltiples de comunicación y fortalecimiento de la capacidad y la organización comunitaria, así como el abordaje de las políticas públicas que inciden sobre las condiciones y recursos que facilitan la actividad física • 


\section{REFERENCIAS}

1. Andersen RE, Wadden TA, Bartlett SJ, Zemel B, Verde TJ, Franckowiak SC. Effects of lifestyle Activity Vs structured aerobic exercise in obese Women: a randomized trial. JAMA, 1999; 281(4): 335- 340.

2. Centers for Disease Control and Prevention Public Health Focus: physical activity and the prevention of coronary Heart disease. MMWR Morb Mortal Wklu Rep 1993; 42: 669-672.

3. Pate RR, Pratt M, Blair SN, Haskell WL, Macera CA, Bouchard C, et al. A recommendation from the Centers for Disease Control and Prevention and the American College of Sports Medicine. JAMA 1995; 273(5): $402-7$.

4. Oded Bar-Or. Juvenile Obesity, Physical Activity, and Lifestyle Changes: Cornerstones for Prevention and Management. The Physician and Sports Medicine 2000; 28: 51-58.

5. Department of Health and Human Services, Atlanta, GA: Centers for Disease Control and Prevention. State Programs in Action: Exemplary Work to Prevent Chronic Disease and Promote Health, 2003. State Programs in Action Exemplary Work to Prevent Chronic Disease and Promote Health 2003. [Internet]. Available at: http://www.cdc.gov/nccdphp /publications/Exemplary. Consultado en abril de 2004.

6. Corbin CB, Dale D, Pangrazi RP. Promoting Physically Active Lifestyles among Youths. JOPERD 1999; 70(8): 26-28.

7. Gómez LF, Espinosa G, Duperly J, Cabrera GA, Gómez OL. Revisión sistemática de intervenciones comunitarias sobre actividad física en grupos específicos. Colomb Med 2002; 33: 162-170.

8. Matsudo V, Matsudo S, Andrade D, Araujo T, Andrade E, de Oliveira LC, et al. Promotion of physical activity in a developing country: the Agita Sao Paulo experience. Public Health Nutr 2002 February; 5(1A): 253-61.

9. U.S.Department of Health and Human Services. Physical Activity and Health: A Report of the Surgeon General. Atlanta: USDHSS/CDC; 1996.

10. McLeroy K, Bibeau D, Steckler A, Glanz K. An Ecological Perspective on Health Promotion Programs. Health Education Quaerterly 1988; 15(4):351378.

11. Mc Caffree J. Physical activity: How much is enough? The American Dietetic Association 2003; 103: 2.

12. Prieto A. Modelo de prevención de la salud, con énfasis en actividad física, para una comunidad estudiantil universitaria. Revista de Salud Pública 2003; 5(3):284-300.

13. Speck B.J. From Exercise to Physical Activity. Holist Nurs Pract 2002; 16(5): 24 $-31$. 